\title{
ANÁLISE COMPARATIVA DE DOIS MÉTODOS PARA OBTENÇÃO DA TEMPERATURA DE SUPERFÍCIE DO AGLOMERADO URBANO DA REGIÃO METROPOLITANA DE CURITIBA A PARTIR DO SENSOR TM/LANDSAT-5
}

\author{
SILVA, Josemar Pereira da - josemar@ufpr.br \\ Dept de Geografia - Universidade Federal do Paraná \\ CANEPARO, Sony Cortese- sony@ufpr.br \\ Dept de Geografia - Universidade Federal do Paraná \\ PAULA, Eduardo Vedor de - edugeo@ufpr.br \\ Dept de Geografia - Universidade Federal do Paraná
}

\begin{abstract}
RESUMO: A estimativa da temperatura da superfície (TS), a partir de sensores remotos térmicos para a delimitação de ilhas de calor de superfície (ICS), possui limitações devido aos efeitos de absorção e espalhamento causados pela atmosfera, no momento da passagem do sensor. Neste sentido, o objetivo deste trabalho é comparar os resultados apresentados pelo método de correção atmosférica proposto por Coll et al. (2010), com o método proposto por Artis e Carnahan (1982), o qual não contempla correção atmosférica para estimativa da TS. Para aplicação destes dois métodos utilizou-se a cena do sensor TM/Landsat-5 (órbita 78 / ponto 200) do dia 19/11/2010, a qual compreende a área do Aglomerado Urbano da Região Metropolitana de Curitiba (AU-RMC). Como suporte para interpretação da TS foi empregado o Índice de Vegetação por Diferença Normalizada (NDVI). A análise dos resultados permitiu observar que $99,5 \%$ dos valores da TS obtidos pelo método com correção atmosférica (MCA) apresentam temperaturas mais elevadas que os obtidos pelo método que não possui correção atmosférica (MSC), sendo que dentro deste universo observado, em mais de $52 \%$ da área estimada a TS superou $2^{\circ} \mathrm{C}$ em relação ao MSC.
\end{abstract}

Palavras-Chave: Sensoriamento Remoto, Infravermelho Termal, Correção Atmosférica, Ilhas de Calor. COMPARATIVE ANALYSIS OF TWO METHODS FOR OBTAINING THE SURFACE TEMPERATURE OF URBAN CLUSTER OF CURITIBA METROPOLITAN REGION FROM THE SENSOR TM / LANDSAT-5

ABSTRACT: The thermal estimate surface temperature (TS) through remote sensors to the delimitation of surface heat islands ( $\mathrm{SHI}$ ) has its limitations due to the effects of absorption and scattering caused by the atmosphere in the moment of the passage of the sensor. Bearing this in mind, this work aims to compare the results presented by the method of atmospheric correction proposed by Coll et al (2010) in contrast with the method proposed by Artis and Carnahan (1982), in which the atmospheric correction is not contemplated to estimate the ST. In order to apply these methods, the scene (captured on November 19, 2010) of the sensor TM/Landsat-5 (orbit 78 / point 200). The area covered was the Urban Agglomerate of Curitiba and its border cities within the Metropolitan Region. In order to reinforce the consistency of the interpretation of the TS data results, the Index of Vegetation by Normalized Difference (NDVI) was implemented.The analysis of the results showed that $99.5 \%$ of the pixel values obtained by the method with atmospheric correction presented more elevated temperatures than the results obtained by the method without atmospheric correction. Within the statistical universe observed, more than $52 \%$ of the TS estimated area had an increase of $2^{\circ} \mathrm{C}$ in relation to the SHI.

Keyword: Remote Sensing, Infrared Thermal, Atmospheric Correction, Heat Islands.

\section{INTRODUÇÃO}

A interação entre a superfície-atmosfera tem papel importante no sistema climático terrestre. Diversos estudos têm abordado os problemas ocasionados pelas alterações decorrentes das atividades humanas como remoção da vegetação, adensamento construtivo e impermeabilização do solo, as quais estão diretamente relacionadas ao recente e acelerado processo de urbanização das cidades brasileiras (STEINKE, 2010; MESQUITA, 2012).

Estas modificações na cobertura da superfície provocam alterações nas condições atmosféricas de uma região, principalmente sobre as áreas urbanas, uma vez que proporcionam mudanças na rugosidade e nas propriedades térmicas da superfície, reduzindo assim a intensidade dos ventos e da umidade do solo (OKE, 1987). 
De acordo com Gartlant (2010), os efeitos supracitados e o aumento do uso de material construtivo acabam repercutindo na redução da evaporação e aumento do armazenamento de calor da superfície urbana. Desta maneira, o calor antropogênico em ambientes urbanos tornase mais elevado que em áreas rurais, devido ao aprisionamento de energia que tem contribuído com o surgimento de ilhas de calor em diferentes escalas.

Para avaliar as modificações causadas na superfície terrestre e sua relação com o aumento da temperatura urbana tem-se empregado sensores térmicos que operam na faixa do infravermelho termal, os quais captam a energia emitida pela superfície terrestre. Desta maneira, o sinal recebido pelo sensor pode ser convertido em temperatura de superfície aparente ou temperatura de brilho.

A partir deste procedimento o uso de imagens térmicas tem sido muito comum nos estudos de clima urbano, tendo como marco inicial no Brasil o trabalho de Lombardo (1985) para identificação de ilhas de calor em São Paulo/SP.

Voogt e Oke (2003) e Weng (2009) observaram em suas pesquisas que o sensoriamento remoto possui grande aplicabilidade nos estudos de clima urbano, uma vez que permite a obtenção dos valores da Temperatura da Superfície (TS) de forma espacializada em diferentes escalas. Nestes estudos, as áreas onde a TS são mais elevadas identificam o surgimento de ilhas de calor de superfície (ICS), onde denotam paisagens mais aquecidas que as áreas circundantes. As áreas onde a TS são mais amenas correspondem as ilhas de frescor de superfície (IFS) e estão atreladas a áreas verdes e de menor adensamento urbano.

Porém, estes autores ressaltam que para a obtenção de melhores resultados na estimativa da TS, por meio de sensoriamento remoto, faz-se necessário o emprego de procedimentos que possibilitem a remoção dos efeitos causados pela absorção e espalhamento da radiação emitida pela superfície para a atmosfera.

Diversos sensores térmicos em plataformas orbitais como AVR, LANDSAT, CBERS e MODIS tem sido empregados para obtenção da TS, como também sensores hiperespectrais acoplados em aeronaves como o HSS (Hyperspectral Scanner System) utilizado por Andrade (2011).

Segundo Mendonça (2003), para a avaliação do campo térmico produzido pelas cidades, enquanto subdivisão do clima urbano tem sido empregado imagens térmicas provenientes sensores orbitais, de maneira especial para o estudo das ilhas de calor. Para este autor, as regiões metropolitanas de grandes dimensões e diversidade espacial, apresentam desafios teóricos e técnicos para o estudo do clima local, principalmente pela diversidade de uso do solo neste ambiente.

No Brasil, diferentes métodos de obtenção de TS a partir dos sensores térmicos da série TM/Landsat-5 e ETM/Landsat-7 têm sido empregados para o estudo do ambiente urbano, como, por exemplo, nos estudos das cidades de Porto Alegre por Collischonn (1998), em Piracicaba por Coltri (2006), em Recife por Moreira (2009) e em Curitiba por Silva (2014).

Para avaliar o ambiente urbano das cidades de São Paulo e Rio de Janeiro, Fuckner (2008) empregou as imagens térmicas provenientes do sensor ASTER/Terra.

Considerando os recentes avanços para obtenção da TS a partir de sensores térmicos a nível orbital, o objetivo deste trabalho consiste na análise comparativa entre o método proposto por Coll et al. (2010) o qual possibilita remoção dos efeitos causados pela atmosfera, com resultado obtido pelo método proposto por Artis e Carnahan (1982) ${ }^{1}$.

\footnotetext{
${ }^{1}$ ARTIS, D. A.; CARNAHAN, W.H. Survey of emissivity variability in thermography of urban áreas. Remote Sensing of Environment, v. 12, n.4, p.313-329, Sept. 1982.
} 
Para aplicação destes métodos, foi adotada a área (Figura 1) que compreende o Aglomerado Urbano da Região Metropolitana de Curitiba (AU-RMC), a qual apresenta uma mancha contínua do adensamento urbano da cidade de Curitiba e sua conurbação com municípios limítrofes.
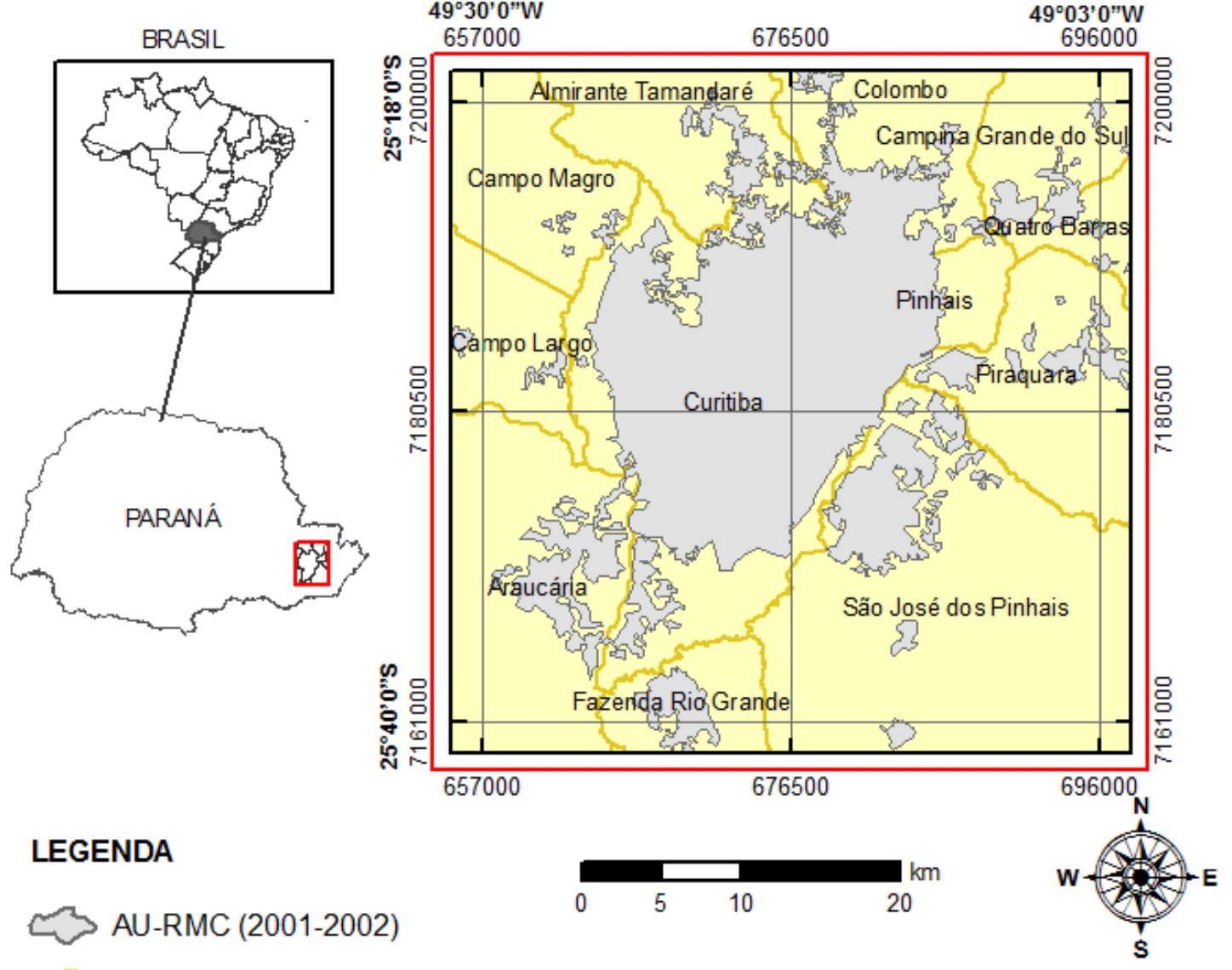

Limites Municipais

Fonte: IBGE, 2005; COMEC, 2005

Figura 1 - Mapa de localização do Aglomerado Urbano de Curitiba (AU-RMC)

\section{MATERIAIS E MÉTODOS}

A comparação entre os dois métodos selecionados de obtenção da TS foi realizada a partir da cena adquirida pelo sensor TM/Landsat-5 (órbita 78/ ponto 200) do dia 19/11/2010, o qual apresentou céu limpo no momento da aquisição da imagem digital. Esta cena foi obtida pelo catálogo online de imagens do Instituto Nacional de Pesquisas Espaciais (INPE).

A Figura 2 apresenta o fluxograma que representa as etapas desenvolvidas no âmbito da presente pesquisa. Sendo que as duas primeiras etapas são comuns para a banda 3 (vermelho), banda 4 (infravermelho próximo), ambas com resolução espacial de 30 metros, e banda 6 (infravermelho térmico) com resolução espacial de 120 metros. 


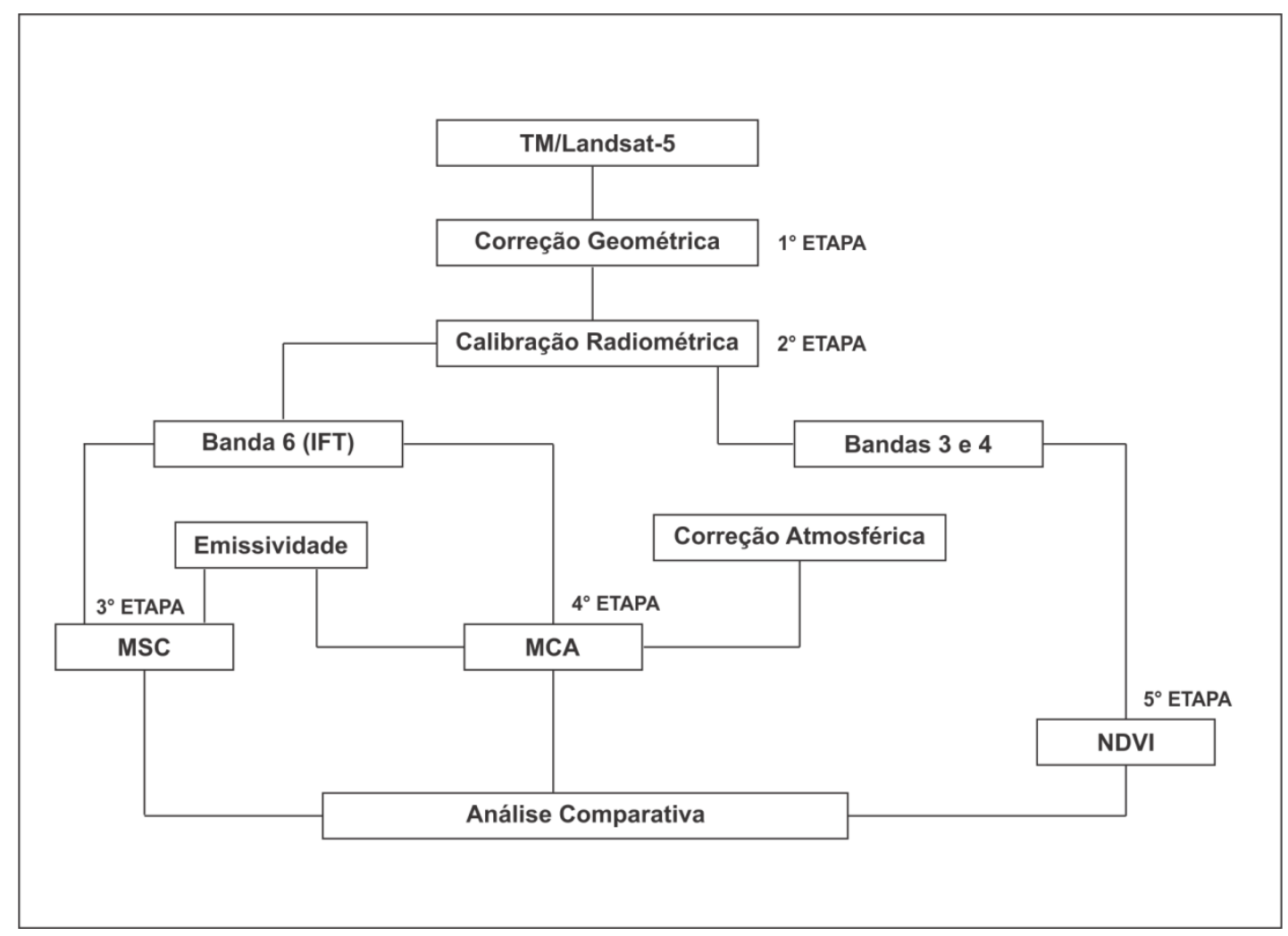

Figura 2 - Fluxograma das etapas de processamento digital e cartografia

Na Etapa 1 foi realizada a correção geométrica da cena escolhida, sendo usada como referência uma imagem ortorretificada ETM/Landsat-7 do ano de 2005, obtida no website da Global Visualization Viewer.

A Etapa 2 consistiu na calibração radiométrica das três bandas empregadas neste estudo, conforme descrição apresentada na Equação 1:

$$
L_{\lambda}=\left(G_{\text {rescale }} * Q_{\text {cal }}\right)+B_{\text {rescale }}
$$

Onde:

$L_{\lambda} \quad=$ radiância em unidade de $\mathrm{W} /\left(\mathrm{m}^{2} . \mathrm{sr} . \mu \mathrm{m}\right) ;$

$G_{\text {rescale }}=$ fator de ganho de redimensionamento específico da banda (W/m².sr. $\left.\mu \mathrm{m}\right) \mathrm{DN}$;

$Q_{c a l}=$ valor do pixel (Número Digital);

$B_{\text {rescale }}($ Offset $)=$ fator de perda de redimensionamento específico da banda $\mathrm{W} /\left(\mathrm{m}^{2} . \mathrm{sr} . \mu \mathrm{m}\right)$.

Os coeficientes de calibração do sensor TM/Landsat-5 utilizados encontra-se descritos no Quadro 1. 


\begin{tabular}{|c|c|c|c|c|c|c|c|}
\hline \multicolumn{6}{|l|}{ Calibração do sensor TM (Qcalmin = 1 and Qcalmax = 255) } \\
\hline Bandas & $\begin{array}{c}\text { Spectral } \\
\text { Range }\end{array}$ & $\begin{array}{c}\text { Center } \\
\text { wavelengh }\end{array}$ & LMINy & LMAXy & $G_{\text {rescale }}$ & $B_{\text {rescale }}$ & $E S U N_{\lambda}$ \\
\hline Unidades & \multicolumn{2}{|c|}{$\mu \mathrm{m}$} & $\mathrm{W} /\left(\mathrm{m}^{2} . \mathrm{sr} . \mu \mathrm{m}\right)$ & $\begin{array}{c}\left(\mathrm{W} / \mathrm{m}^{2}\right. \\
. \mathrm{sr} . \mu \mathrm{m} / \\
\mathrm{DN}\end{array}$ & $\begin{array}{c}\mathrm{W} /\left(\mathrm{m}^{2}\right. \\
. \mathrm{sr} . \mu \mathrm{m})\end{array}$ & $\mathrm{W} /\left(\mathrm{m}^{2} . \mu \mathrm{m}\right)$ \\
\hline 3 & $\begin{array}{c}0,626- \\
0,693\end{array}$ & 0,660 & $-1,17$ & 264 & 1,043976 & $-2,21$ & 1536 \\
\hline 4 & $\begin{array}{c}0,776- \\
0,904\end{array}$ & 0,840 & $-1,51$ & 221 & 0,876024 & $-2,39$ & 1031 \\
\hline 6 & $\begin{array}{c}10,45- \\
12,42\end{array}$ & 11,435 & 1,2378 & 15,3032 & 0,055376 & 1,18 & N/A \\
\hline
\end{tabular}

Quadro 1 - Coeficientes de calibração radiométrica para o TM/Landsat-5 Fonte: CHANDER et al. (2009)

Finalizado os procedimentos de correção geométrica e calibração radiométrica que são comuns para as bandas 3, 4 e 6, na Etapa 3 aplicou-se o método proposto por Artis e Carnahan (1982) para obtenção da TS, denominado neste trabalho como Método Sem Correção (MSC).

O primeiro procedimento realizado para o MSC, consistiu na obtenção da temperatura de brilho, descrito pela Equação 2:

$$
T_{b}=\frac{K 2}{\ln \left(\frac{K 1}{L_{\lambda}}+1\right)}
$$

onde:

$T_{b} \quad=$ temperatura de brilho;

K2 = constante de calibração de pré-lançamento (Kelvin);

$K 1=$ constante de calibração de pré-lançamento em $\mathrm{W} /\left(\mathrm{m}^{2} . \mathrm{sr} . \mu \mathrm{m}\right)$;

$L_{\lambda} \quad=$ radiância em unidade em $\mathrm{W} /\left(\mathrm{m}^{2}\right.$.sr. $\left.\mu \mathrm{m}\right) ;$

$\ln \quad=$ logaritmo natural.

De acordo com Chander et al. (2009) as constantes de calibração para a banda térmica do sensor TM/Landsat-5 são $\mathrm{K} 1=607.76\left(\mathrm{~W} /\left(\mathrm{m}^{2} \mathrm{sr}\right.\right.$ um); K2 = $1260.56(\mathrm{~K})$. 
A partir da obtenção da $T_{b}$, o procedimento seguinte do MSC consistiu na obtenção da TS a partir da Equação 3:

$$
T_{s}=\frac{T_{b}}{1+\left(\frac{\lambda_{6} T_{b}}{a}\right) \ln \varepsilon}
$$

onde:

$T_{s} \quad$ = temperatura da superfície;

$T_{b} \quad=$ temperatura de brilho do sensor termal;

$\lambda_{6}=$ comprimento de onda médio da radiância da banda $6(11,435 \mu \mathrm{m})$;

$a=\mathrm{hc} / \sigma\left(1.438 \times 10^{-2} \mathrm{mK}\right) ;$

$\ln \varepsilon \quad=$ logaritmo natural da emissividade da superfície.

Sendo que:

$\mathrm{h}=$ constante de Planck $\left(6.28 \times 10^{-34} \mathrm{~J} / \mathrm{seg}\right)$;

$\mathrm{c}=$ velocidade da luz $\left(2.998 \times 10^{8} \mathrm{~m} / \mathrm{seg}\right)$;

$\sigma=$ constante de Stefan-Bolzmann $\left(1.38 \times 10^{-23} \mathrm{~J} / \mathrm{K}\right)$;

Dependendo do sensor remoto empregado, a obtenção da TS envolve uma complexidade extensa de correções, por meio de modelos empíricos e por emprego de códigos de transferência radiativa (QIN et al., 2001; SOBRINO et al., 2004; VOOGT e OKE, 2003; SOUZA, 2008; WENG, 2009).

Nestas correções são empregados os valores da emissividade da superfície terrestre (obtida por radiômetros portáteis e/ou sensores remotos) e dados das condições atmosféricas por meio de radiossondas (balões atmosféricos), ou por dados das estações meteorológicas no momento da passagem do sensor.

A escolha da Equação 3 foi motivada por esta permitir a inserção da variável emissividade $(\varepsilon)$ da superfície no seu cômputo.

Para os dois métodos, optou-se em utilizar o valor de emissividade de $(0,92)$, o qual corresponde ao valor médio das superfícies urbanas, conforme proposto inicialmente por Nichol (1994) e adotado por Collischonn (1998) e Steinke (2004).

O método proposto por Coll et al. (2010), denominado neste trabalho como Método com Correção Atmosférica (MCA) que será descrito a seguir, na Etapa 4, corresponde ao conjunto de procedimentos que possibilitam a remoção efeitos causados pela atmosfera para obtenção da radiância emergente que é registrada pelo sensor TM/Landsat-5.

A aplicação deste método foi subsidiada pelo uso da Calculadora de Correção Atmosférica (WEBCALC), para o sensor TM/Landsat-5, a qual possui acesso livre pelo endereço eletrônico http://atmcorr.gsfc.nasa.gov. 
A WEBCALC possibilita a obtenção dos dados de transmissividade atmosférica, radiância emitida e recebida pela superfície. Para a obtenção destes valores foram utilizadas as condições meteorológicas da superfície (temperatura do ar, pressão e umidade relativa) no momento da passagem do sensor, bem como os dados de localização (altitude, latitude e longitude) da área de estudo.

Estes dados foram obtidos pela Estação Meteorológica do Sistema Meteorológico do Paraná (SIMEPAR), localizada no Centro Politécnico da Universidade Federal do Paraná (UFPR), sendo relativos ao dia 19/11/2010 (10 horas da manhã).

A partir destes dados, foram simulados os valores de $L \uparrow=$ radiação emitida pela superfície, $L \downarrow=$ radiação em ondas longas que atinge a superfície e $\tau=$ transmissividade da atmosfera pela WEBCALC, cujos resultados são descritos a seguir:
$L \uparrow=1.15 \mathrm{~W} /\left(\mathrm{m}^{2} . \mu \mathrm{m}\right) ;$
$L \downarrow=1.19 \mathrm{~W} /\left(\mathrm{m}^{2} \cdot \mu \mathrm{m}\right) ;$
$\tau \quad=0.85$

Com base nos resultados obtidos pela WEBCALC, para obtenção do valor da radiância espectral considerando a remoção dos efeitos da atmosfera, adotou-se o método proposto por Coll et al. (2010), descrito pela Equação 4:

$$
C V_{R}=\frac{L_{\lambda}-L \uparrow}{\varepsilon \tau}-\frac{1-\varepsilon}{\varepsilon} L \downarrow
$$

onde:

$C V_{R}=$ radiância com correção atmosférica;

$L_{\lambda}=$ valor da radiância do sensor;

$\varepsilon=$ emissividade da superfície.

O procedimento seguinte do MCA, apresentado por Coll et. al. (2010) consiste na obtenção TS pela Equação 5:

$$
T_{S}=\frac{K 2}{\ln \left(\frac{K 1}{C V_{R}}+1\right)}
$$

onde: 
$T_{S} \quad$ = temperatura da superfície;

$K 2$ = constante de calibração de pré-lançamento (Kelvin);

$K 1=$ constante de calibração de pré-lançamento em $\mathrm{W} /\left(\mathrm{m}^{2} . \mu \mathrm{m}\right)$;

$C V_{R}=$ radiância com correção atmosférica.

Nos dois métodos aplicou-se a conversão dos valores da TS obtidos de Kelvin para graus Celsius $\left({ }^{\circ} \mathrm{C}\right)$.

A forma como a superfície é ocupada influencia a quantidade de energia que pode ser armazenada e emitida em forma de calor (Gartland, 2010).

Neste sentido, para verificar as condições da cobertura da superfície no momento da passagem do sensor (Etapa 5) optou-se em utilizar o Índice de Vegetação por Diferença Normalizada (NDVI), descrito na Equação 6:

$$
N D V I=\frac{\left(\rho_{4}-\rho_{3}\right)}{\left(\rho_{4}+\rho_{3}\right)}
$$

onde:

$$
\begin{aligned}
& \rho_{4}=\text { radiância do infravermelho próxima (banda 4) } \\
& \rho_{3}=\text { radiância do vermelho (banda } 3 \text { ) }
\end{aligned}
$$

A classificação da superfície do AU-RMC por meio do NDVI permite a identificação da intensidade ocupada pela vegetação, diferenciando assim as áreas arborizadas, pouco arborizadas e campos. Além disso, é possível distinguir por este procedimento as áreas que não possuem vegetação, obtendo dessa maneira o contraste térmico emitido pela superfície do AU-RMC.

Para este estudo optou-se pelo uso do NDVI, como método de auxílio visual, para análise áreas onde ocorreu maior diferença entre os dois métodos. Devido a diferença entre as escalas espaciais das bandas 3 e 4 com a banda 6 , os produtos gerados não foram sobrepostos.

Conforme interpretação visual, adotou-se as classes de NDVI descritas a seguir:

SV - Área Sem Vegetação;

0 a 0,1 - Limiar entre pouca e sem vegetação;

0,1 a 0,3 - Pouca vegetação;

0,3 a 0,5 - Presença de vegetação;

0,5 a 1 - Maior presença de vegetação.

Por fim, deve-se ressaltar que para a delimitação da área de estudo foram utilizados os arquivos em formato shapefile da Mancha Urbana da RMC elaborado pela COMEC (2005) e arquivo de Limites municipais do Paraná elaborado por IBGE (2005). 


\section{RESULTADOS E DISCUSSÕES}

A Figura 3 apresenta a distribuição espacial e histogramas correspondentes da TS obtida para os dois métodos analisados, respectivamente MSC (A) e MCA (B).

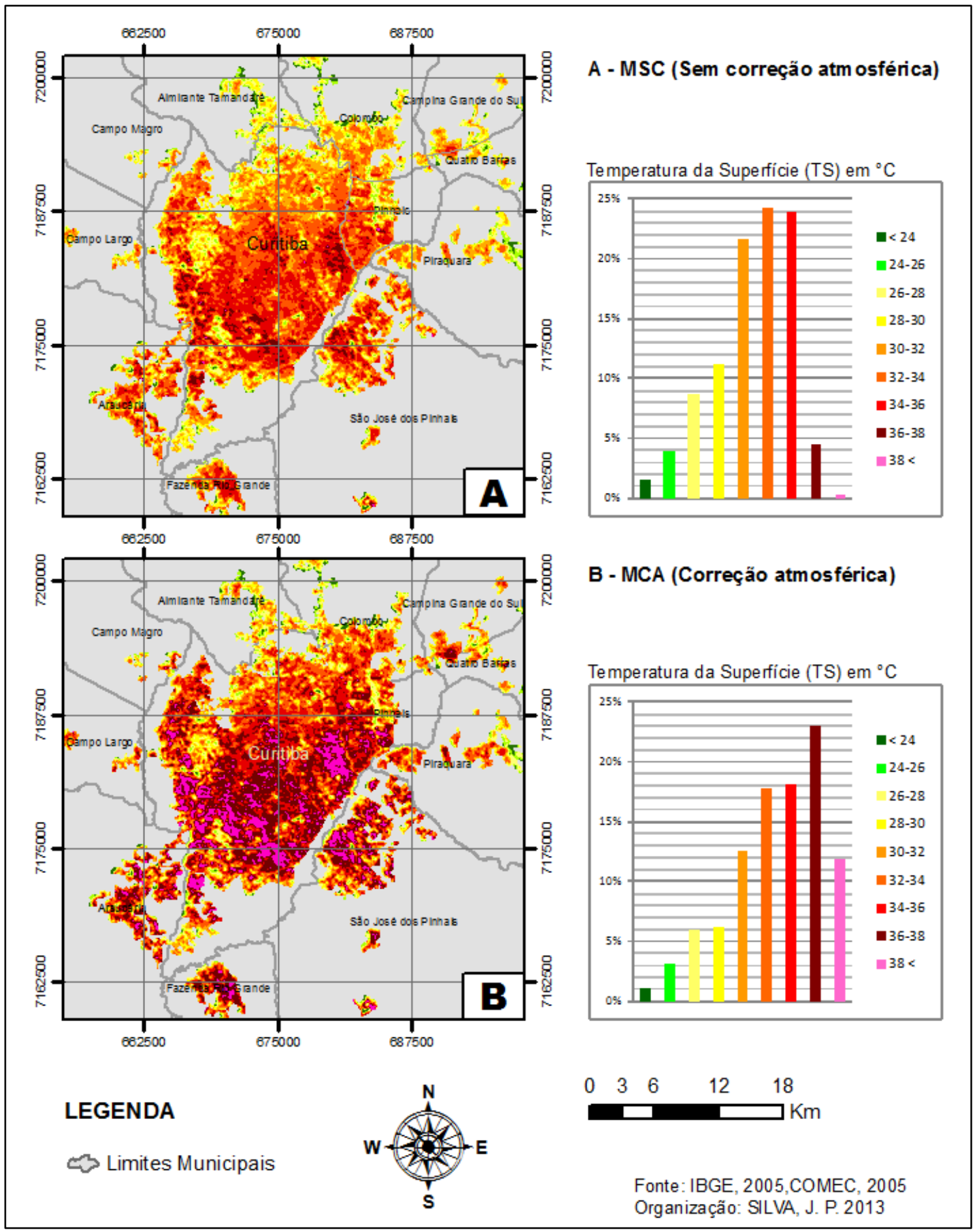

Figura 3 - Item A (MSC - Método Sem Correção), item B (MCA - Método com Correção Atmosférica)

O MSC (Figura 3A) apresenta TS mais baixas que as obtidas pelo MCA (Figura 3B). Este fato está diretamente relacionado à remoção dos efeitos atmosféricos pelo MCA, onde por meio de 
simulação da WEBCALC, são obtidos os parâmetros que atenuam a radiância emitida pela superfície.

Deste modo, os valores dos números digitais originais de cada pixel (de 0 a 255) que compõem a imagem térmica ao serem corrigidos por estes parâmetros e depois convertidos em valores de TS são reamostrados automaticamente em classes distintas pelo MCA, ou seja, ocorre uma melhora teórica da radiância emitida pela superfície em relação ao MSC.

Ao se considerar os valores obtidos pelos dois métodos, verifica-se que 99,5\% da área do AURMC pelo MCA possui TS superiores ao MSC. Assim, observa-se nos histogramas da Figura 3 que o procedimento de correção atmosférica do MCA resultou na dispersão dos pixels para valores de TS mais elevados que os obtidos pelo MSC.

Na Tabela 1 apresenta-se a comparação estatística entre os dois métodos. O resultado da TS para o MCA denotou a maior amplitude térmica para a área do AU-RMC, sendo correspondente a $50,68^{\circ} \mathrm{C}$, que é $8,89^{\circ} \mathrm{C}$ superior ao valor apresentado pelo MSC.

\begin{tabular}{c|c|c|c|c|c}
\hline MÉTODO & $\begin{array}{c}\text { MÍNIMO } \\
\left({ }^{\circ} \mathbf{C}\right)\end{array}$ & $\begin{array}{c}\text { MÉDIA } \\
\left({ }^{\circ} \mathbf{C}\right)\end{array}$ & $\begin{array}{c}\text { MÁXIMA } \\
\left({ }^{\circ} \mathbf{C}\right)\end{array}$ & $\begin{array}{c}\text { AMPLITUDE } \\
\text { TÉRMICA } \\
\left({ }^{\circ} \mathbf{C}\right)\end{array}$ & $\begin{array}{c}\text { DESVIO } \\
\text { PADRÃO } \\
\left({ }^{\circ} \mathbf{C}\right)\end{array}$ \\
\hline MSC (A) & 6,73 & 31,82 & 48,52 & $41,79^{\circ} \mathrm{C}$ & 3,21 \\
MCA (B) & 2,92 & 33,7 & 53,6 & $50,68^{\circ} \mathrm{C}$ & 3,85 \\
Diferença & $-3,8$ & 1,93 & 5,12 & $8,89^{\circ} \mathrm{C}$ & 0,65
\end{tabular}

Tabela 1 - Comparativo entre os valores da TS obtidos para o MSC e MCA Fonte: Autor (2014)

Conforme os resultados apresentados no histograma do MSC (Figura 3A), nota-se que a maior parcela dos pixels, ou seja, cerca de $70 \%$ da TS concentra-se na faixa de valores entre 30 a $36^{\circ} \mathrm{C}$ para o MSC, o que é diferente do resultado obtido pelo MCA (Figura 3B), no qual esta mesma faixa compreende apenas $49 \%$ da TS estimada para o AU-RMC.

Observa-se que a diferença entre as duas faixas ocorreu devido à uma melhora do número digital da imagem original para um gradiente mais elevado decorrente da correção radiométrica pelo MCA. Desta maneira, a faixa superior a $36^{\circ} \mathrm{C}$ representa $35 \%$ para o MCA, enquanto que no $\mathrm{MSC}$ as TS superiores a $36^{\circ} \mathrm{C}$ não passam de $5 \%$.

Os resultados apresentados na Figura 3 evidenciam que as maiores temperaturas do AU-RMC ocorrem nas mesmas áreas, porém seus valores são mais elevados no MCA. Este fato pode ser atribuído ao refinamento do valor do pixel bruto, conforme o valor de correção sobre a radiância térmica obtida pelo MCA.

As manchas, representadas em tons de rosa (Figura 3B) estão relacionadas principalmente às áreas mais adensadas do AU-RMC, com ausência e/ou menor quantidade de vegetação, sendo caracterizadas por áreas densamente edificadas, constituídas de superfícies lisas como de telhados e estacionamentos de supermercados, barracões e indústrias.

Com o intuito de comparar os valores obtidos pelos métodos MSC e MCA, foi elaborada a Figura 4A, que corresponde à subtração dos valores do mapa temático do MCA sobre o mapa do MSC. 


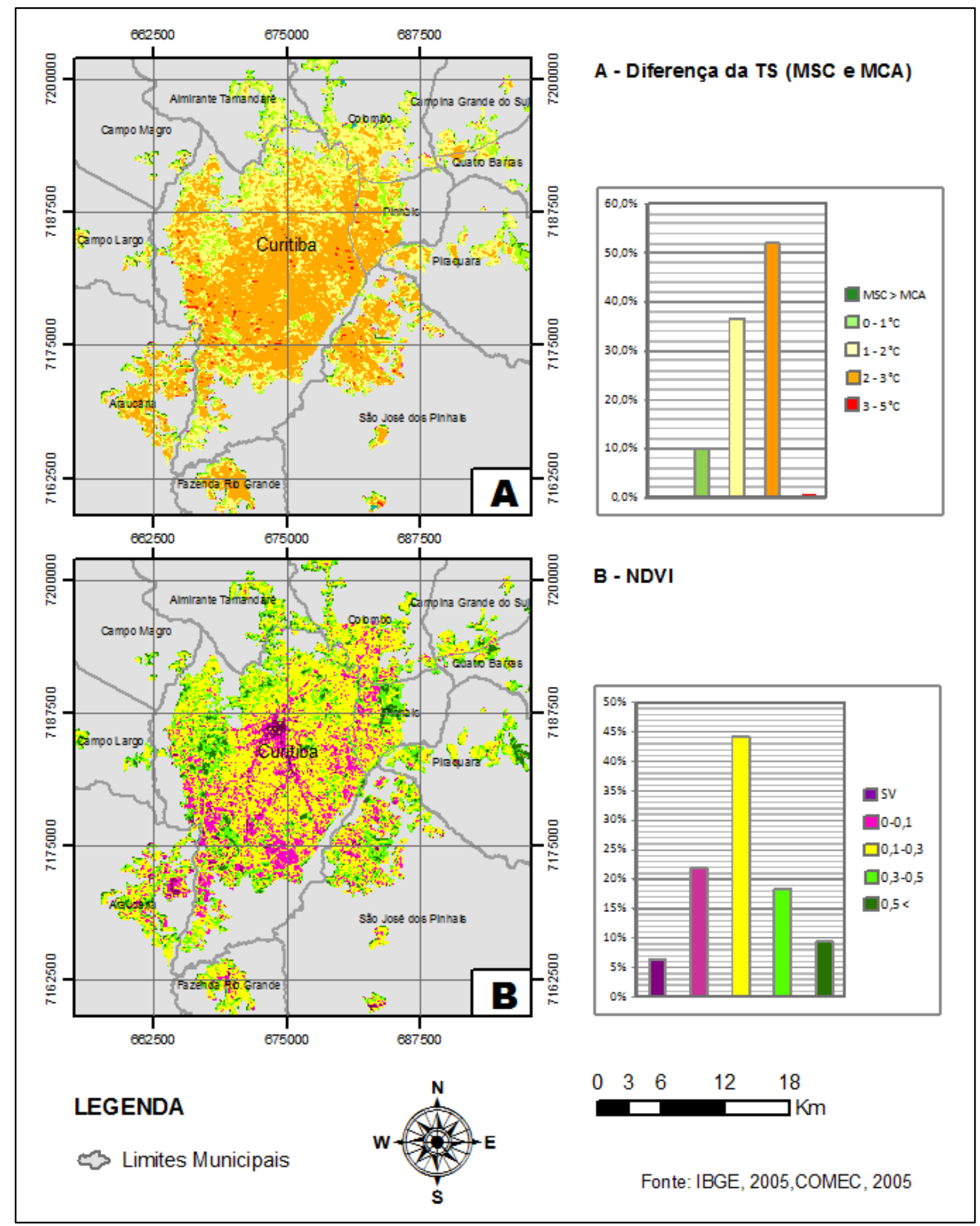

Figura 4 - Item A (Subtração dos valores do MCA sobre o MSC), item B (Cobertura da superfície conforme a quantidade de vegetação)

A região central do AU-RMC concentra a maior diferença da TS em área (52\%) para os dois métodos, onde a TS é de 2 a $3^{\circ} \mathrm{C}$. Estas áreas correspondem às superfícies com limiares de NDVI entre 0 a 0,3, ou seja, pouca ou até mesmo sem cobertura vegetal (SV) na Figura 4B.

A partir da comparação visual entre a subtração dos métodos MSC e MCA (Figura 4A) com o NDVI da superfície do AU-RMC (Figura 4B), observa-se que as menores diferenças térmicas entre os dois métodos correspondem principalmente às áreas onde a classe de NDVI é superior a 0,3 , ou seja, presença de cobertura vegetal. 
Além disso, para os dois métodos as áreas do AU-RMC que possuem cobertura vegetal, ou seja, onde o valor de NDVI é superior a 0,3 também apresentam as TS mais baixas. A diferença da TS entre 3 a $5^{\circ} \mathrm{C}$ para o MSC e MCA correspondem espacialmente as classes de NDVI de SV a $0,1$.

\section{CONCLUSÃO}

Com base nos resultados apresentados, os parâmetros atmosféricos obtidos por meio de simulação das condições de superfície (temperatura do ar, umidade e pressão atmosférica) e de sua localização (altitude, latitude e longitude) empregados pelo MCA, proporcionaram TS mais elevadas que as obtidas pelo MSC.

Os dois métodos avaliados neste trabalho possibilitam a espacialização da TS do AU-RMC, as quais podem subsidiar diferentes estudos aplicados ao clima urbano como, por exemplo, a caracterização de ICS.

No entanto, ressalta-se que devido à heterogeneidade da superfície urbana, a maioria dos estudos sugere que a correção atmosférica, utilizada pelo MCA, seja aplicada apenas para regiões de pequena escala, considerando assim a emissividade local e a temperatura do ar isotermicamente homogênea próxima da superfície.

Para os dois métodos, as TS mais baixas coincidem com as superfícies onde o NDVI é mais elevado, ou seja, maior cobertura vegetal.

Em ambos os métodos as TS mais elevadas se situam principalmente nas áreas mais adensadas do AU-RMC.

Apesar de simples, o MSC permite o melhoramento do valor da TS, considerando os ajustes da equação em relação a simples conversão do número digital do sensor em temperatura de brilho.

Entretanto, melhores resultados para os dois métodos podem ser obtidos a partir do detalhamento das coberturas intraurbanas, ou seja, considerando os distintos valores de emissividade da superfície e sua relação com a quantidade de insolação.

Neste sentido, ressalta-se que em trabalhos futuros os valores obtidos da TS possam ser validados no momento da passagem do sensor remoto com dados amostrais representativos da cobertura da superfície urbana por meio de sensores térmicos portáteis, permitindo assim comparar estes resultados com os obtidos pelos dois métodos.

\section{REFERÊNCIAS}

ANDRADE, L.L. Estimativa da temperatura e emissividade com imagens do sensor HSS (Hyperspectral Scanner System) e suas relações com materiais urbanos. 128 p. Dissertação (Mestrado em Sensoriamento Remoto) - INPE, São José dos Campos, 2011.

CHANDER, G; MARKHAM, B; HELDER, D.L. Sumary of current radiometric calibration coefficients for Landsat MSS, TM, ETM+, and EO-1 ALI sensors. Remote Sensing of Environment. V .113. p.893-903. 2009.

COLL, C.; GALVE, J.M.; SÁNCHEZ, J.M.; CASELLES, V. Validation of Landsat-7/ETM+ ThermalBand Calibration and Atmospheric Correction With Ground-Based Measurements. IEEE Transactions on Geoscience and Remote Sensing, v 48, $n^{\circ} 1$, January, 2010.

COLLISCHONN, E. O campo térmico da Região Metropolitana de Porto Alegre: Uma Análise a partir da Interação das Variáveis Ambientais na Definição do Clima Local. $155 f$. 
Dissertação (Mestrado em Geografia) - Universidade Federal de Santa Catarina, Florianópolis, 1998.

COLTRI, P. Influência do uso e cobertura do solo no clima de Piracicaba, São Paulo: análise de séries históricas, ilhas de calor e técnicas de sensoriamento remoto. $166 \mathrm{f}$. Dissertação (Mestrado em Agronomia) - Escola Superior de Agricultura Luiz de Queiroz, Universidade de São Paulo, Piracicaba, 2006.

FUCKNER, M.A. Aplicação de Imagens ASTER no Estudo do Ambiente Urbano de São Paulo e Rio de Janeiro. 2007. 230 p. (INPE-15203-TDI/1303). Dissertação (Mestrado em Sensoriamento Remoto) - Instituto Nacional de Pesquisas Espaciais, São José dos Campos, 2008.

GARTLAND, L. Ilhas de Calor: Como mitigar zonas de calor em áreas urbanas. Tradução de Silvia Helena Gonçalves. São Paulo: Oficina de Textos, 2010. 223p.

IPPUC - Instituto de Pesquisa e Planejamento Urbano de Curitiba. Curitiba em dados. Disponível em: <http://ippucnet.ippuc.org.br > Acessado em: 13 de junho 2012.

ITCG - Instituto de Terras, Cartografia e Geociências.

Disponível em: <http://www.itcg.pr.gov.br > Acessado em: 13 de junho 2012.

LOMBARDO, M.A. Ilha de calor nas metrópoles: o exemplo de São Paulo. São Paulo: Hucitec, 1985. 244p.

MENDONÇA, F; DUBREUIL, V. Termografia de superfície e temperatura do ar na RMC (região metropolitana de Curitiba/PR). R. RA'E GA. n. 9, p. 25-35, 2005.

MESQUITA, F.L.L. Monitoramento do balanço de radiação na região metropolitana do Rio de Janeiro. 2012. 73f. Dissertação (Mestrado em Meteorologia) - PPGM-IGEO-CCMN, Universidade Federal do Rio de Janeiro, Rio de Janeiro, 2012.

MOREIRA, E.B.M. Variação espacial e multitemporal das temperaturas à superfície na cidade do Recife. Recife, 2009, 98p. Dissertação (Mestrado em Geografia) - Departamento de Ciências Geográficas - Coordenação de Pós - Graduação em Geografia.

MONTEIRO, C.A.F; MENDONÇA, F. A.; (Orgs.). Clima Urbano. São Paulo-SP: Contexto, 2003. 192 p.

NICHOL, J.E. A GIS-Based Approach to Microclimate Monitoring in Singapore's High-Rise Housing Estates. Photogrammetric Engineering \& Remote Sensing, v.60, n. 10, p. 12251232, 1994.

OKE, T.R. Boundary Layer Climates. 2 ed. Methuen, 1987. 289 p.

QIN, Z., KARNIELI, A.; BERLINER P. A mono-window algorithm for retrieving land surface temperature from Landsat TM data and its application to the Israel-Egypt border region. International Journal of Remote Sensing, 22, 3719 - 3746, 2001.

SAYDELLES, A.P. Estudo do campo térmico e das ilhas de calor urbano em Santa Maria. 2005, 219f. Dissertação (Mestrado em Geografia) - Universidade Federal de Santa Maria, Santa Maria, 2005.

SILVA, J. P. Análise do comportamento térmico da superfície do Aglomerado Urbano da região metropolitana de Curitiba durante os anos de 1985 a 2010 a partir do satélite Landsat-5. 169 f. Dissertação (Mestrado em Geografia) Universidade Federal do Paraná, 2014. 
SOBRINO, J. A.; JIMENEZ-MUÑOZ, J. C.; PAOLINI, L.: Land surface temperature retrieval from LANDSAT TM 5. Remote Sensing of Environment, 90, 434 - 440, 2004.

SOUZA, J. D.. Modelo Físico-Matemático de correção atmosférica para imagens TMLandsat 5 MODIS-TERRA/AQUA. Tese de Doutorado, CCT/UFPb, Campina Grande, Pb, 2008, 177p.

STEINKE, V.A; STEINKE, E.T. ; SAITO, C. H. Estimativa da temperatura de superfície em áreas urbanas em processo de consolidação: reflexões e experimentos em Planaltina - DF. Revista brasileira de climatologia, v. 6, p. 37/56, 2010.

VOOGT, J.A.; OKE, T.R. Thermal remote sensing of urban climates. Remote Sensing of Environment. Oxford, v.86, p.370-384, 2003.

WENG, Q.; LU, D.; SCHUBRING, J. Estimation of land surface temperature - vegetation abundance relationship for urban heat island studies. Remote Sensing of Environment. Oxford, v.89, p.467-483. 2004.

WENG, Q. Thermal infrared remote sensing for urban climate and environmental studies: Methods, applications, and trends. ISPRS Journal of Photogrammetry and Remote Sensing. 64, p. 335_344. 2009.

XIAN, G.; CRANE, M. An analysis of urban thermal characteristics and associated land cover in Tampa Bay and Las Vegas using Landsat satellite data. Remote Sensing of Environment. Oxford, v.104, p.147-156. 2006. 